\title{
A Tale of two derivatives: Phase space symmetries and Noether charges in diffeomorphism invariant theories
}

\author{
Brian P. Dolan \\ Department of Theoretical Physics, Maynooth University, Maynooth, Co. Kildare, Ireland \\ and School of Theoretical Physics, Dublin Institute for Advanced Studies, \\ 10 Burlington Road, Dublin, Ireland
}

(Received 8 May 2018; published 3 August 2018)

\begin{abstract}
For a field theory that is invariant under diffeomorphisms there is a subtle interplay between symmetries, conservation laws and the phase space of the theory. The natural language for describing these ideas is that of differential forms, and both differential forms on space-time and differential forms on the infinite dimensional space of solutions of the equations of motion of the field theory play an important role. There are exterior derivatives on both spaces, and together they weave a double differential complex which captures the cohomology of the theory. This is important in the definition of invariants in general relativity, such as mass and angular momentum and is also relevant to the study of quantum anomalies in gauge theories. We derive the structure of this double complex and show how it relates to conserved quantities in gravitational theories. One consequence of the construction is that conserved quantities can be calculated exactly at finite distance-e.g., it is not necessary to go to asymptotic regimes to calculate the mass or angular momentum of a stationary solution of Einstein's equations; they can be obtained exactly by an integration over any sphere outside the mass even at finite radius.
\end{abstract}

DOI: $10.1103 /$ PhysRevD.98.044009

\section{INTRODUCTION}

Conserved quantities are a consequence of Noether's theorem. The simplest version of Noether's theorem assumes that the Lagrangian governing the dynamics is invariant under some symmetry operation. Under a general variation of the fields $F^{I}$ the variation of the Lagrangian density $L(F)$, viewed as a differential form in an $(n+1)$ dimensional space-time $\mathcal{M}$, is

$$
\delta L\left(F^{I}\right)=E_{J}\left(F^{I}\right) \delta F^{J}+d \theta
$$

where $E_{I}\left(F^{I}\right)=0$ are the equations of motion and $\theta\left(F^{I}, \delta F^{I}\right)$ is an $n$-form which depends on the fields and their variation. When there is symmetry of the dynamics Noether's theorem gives an associated conserved charge. The simplest case is when $L$ itself is an invariant of the symmetry. If $\delta_{\mathcal{Q}}=\epsilon \mathcal{T}_{\mathcal{Q}}$ and $\delta_{\mathcal{Q}} L=0$, where $\mathcal{T}_{\mathcal{Q}}$ is a symmetry generator with an associated charge $\mathcal{Q}$, then

$$
\delta_{\mathcal{Q}} L=d \theta\left(S^{I}, \delta_{\mathcal{Q}} S^{I}\right)=\epsilon d \theta\left(S^{I}, \mathcal{T}_{\mathcal{Q}} S^{I}\right)=0
$$

where $S^{I}$ is a solution of the equations of motion. The usual Noether current associated with the conserved charge $\mathcal{Q}$ is the Hodge dual of $\theta\left(S^{I}, \mathcal{T}_{\mathcal{Q}} S^{I}\right)$

\footnotetext{
*bdolan@thphys.nuim.ie
}

$$
\theta=* j
$$

and it is common in field theory to express the conservation of the Noether current as a zero divergence condition,

$$
d * j=0
$$

Integrating over a region of space-time $\mathcal{M}$ bounded by spacelike hypersurfaces $\Sigma$ and $\Sigma^{\prime}$ gives ${ }^{1}$

$$
\int_{\mathcal{M}} d \theta=\int_{\Sigma} \theta-\int_{\Sigma^{\prime}} \theta=0
$$

Thus the charge

$$
\mathcal{Q}=\int_{\Sigma} * j=\int_{\Sigma^{\prime}} * j
$$

associated with the symmetry and the chosen solution is an invariant-independent of the spacelike hypersurface on which it is evaluated. With Maxwell's equations, e.g.,

$$
d * F=4 \pi * j,
$$

\footnotetext{
${ }^{1}$ If $\Sigma$ and $\Sigma^{\prime}$ have boundaries the fields are assumed to vanish sufficiently fast there that there is no contribution from any timelike component of $\partial \mathcal{M}$.
} 
so $* j$ is exact when the equations of motion are satisfied and the electric charge is obtained from Gauss' law,

$$
\mathcal{Q}=\frac{1}{4 \pi} \int_{\Sigma} d * F=\frac{1}{4 \pi} \int_{\partial \Sigma} * F,
$$

where $\partial \Sigma$ is the boundary of $\Sigma$, taken to envelop all the charges.

Under diffeomorphisms $L$ itself is not invariant; it changes by a surface term, and it is the action that is invariant, not the Lagrangian density. Conserved currents can be defined using the energy-momentum tensor $T_{a b}$ : when $\vec{K}$ is a Killing vector $j^{a}=T^{a}{ }_{b} K^{b}$ is a conserved current. This requires splitting $L$ into a "geometrical" part, such as the Einstein Lagrangian, and a "matter" part from which $T_{a b}$ is derived.

Things get more interesting when a classical symmetry is broken quantum mechanically and we have a quantum anomaly. The Stora-Zumino descent equations then provide a powerful tool for classifying and understanding anomalies [1], and quantum anomalies can break diffeomorphism invariance, or equivalently local Lorentz invariance [2].

The full story goes much deeper however and is related to covariant symplectic structures on the phase space of the theory. It transpires that $\theta$ is a 1 -form on the cotangent bundle $T^{*} \mathcal{S}$ of the space of solutions $\mathcal{S}$ and is related to a symplectic potential associated with the phase space of the dynamical theory.

In this work it will be shown how all of these ideas fit into the same mathematical structure of differential complexes and that the relevant conserved quantities are related to cohomology classes. Among the key ingredients are a general coordinate invariant action and a solution of the equations of motion with a Killing vector generating the symmetry.

Some years ago Crnković and Witten [3] gave a method for constructing a symplectic form on the space of solutions of the equations of motion of a relativistic field theory. They used their formalism to obtain the appropriate symplectic forms for Yang-Mills theory and for general relativity in four dimensions. Their construction provides a covariant description of relativistic field theories in the phase space of solutions modulo gauge transformations and diffeomorhpisms $\mathcal{G}, \hat{\mathcal{S}}=\mathcal{S} / \mathcal{G}$, which is ideally suited to studying symmetries and conserved quantities. The idea of a symplectic structure for diffeomorphism invariant theories was first introduced in [4] to investigate instabilities in rotating relativistic fluids. Wald and Collaborators have generalized Crnković and Witten's formalism to a very wide class of diffeomorphism invariant theories in [5-8] and studied conserved quantities associated with Killing symmetries, such as angular momentum in rotationally invariant solutions and mass in stationary solutions.
This formalism was shown in [8] to reproduce the ADM mass [9] for stationary asymptotically flat black holes in Einstein gravity. At the same time it clarifies the origin of the mysterious factor of 2 that is well-known to arise when comparing the Komar mass with the ADM mass $[10,11]$.

It is shown elsewhere [12] that the Brown-York mass [13] also has a natural interpretation within the framework of Lee and Wald's formalism [5]. The Brown-York mass is defined in terms of the difference of the extrinsic curvature of the sphere at infinity for a given solution and the extrinsic curvature of the sphere at infinity in flat spacetime. Being the difference of two solutions of the equations of motion this is a 1-form on $\mathcal{S}$. It can also be shown [12] that Lee and Wald's formalism reproduces the Bondi mass [14] in stationary space-times.

The construction in [5] is general enough to include theories with a cosmological constant $\Lambda$, of either sign when $\Sigma$ is compact without boundary. When $\Sigma$ has a boundary one restricts to negative $\Lambda$ so that the asymptotic regime of a black hole solution is well-defined. Lee and Wald's expression then agrees with the variation of the Henneaux and Teitelboim mass for asymptotically anti-de Sitter (AdS) Kerr black holes in four-dimensions $[15,16]$. It is not immediately obvious that the Wald and HenneauxTeitelboim masses are the same, the details of the calculation are nontrivial and are given elsewhere $[17,18]$.

\section{THE SYMPLECTIC FORM ON THE SPACE OF SOLUTIONS}

In this section we review Wald's original construction of the symplectic structure on the space of solutions and the associated Noether charge arising from diffeomorphism invariance and show how it is described by a double complex structure. The analysis is general enough to include gauge theories and general diffeomorphic invariant theories of gravity, both in metric formulations and in terms of local Lorentz frames, vielbeins and connections.

Let $\mathcal{F}$ be the space of fields and $\mathcal{S}$ the space of solutions and denote the fields by $F^{I} \subset \mathcal{F}$, indexed by $I$. For simplicity we shall assume that all the fields, including the metric, are dynamical - the situation when the metric is nondynamical is discussed in [8].

A solution of the equations of motion will be denoted by $S^{I} \subset \mathcal{S}$. We shall primarily be interested in field configurations which solve the equations of motion, and functionals will depend not only on the fields $S^{I}$ (primary fields) but also on their partial derivatives of order $k, \partial_{k} S^{I_{k}}$ (descendants), indexed by $I_{k}$.

Consider an action which is an integral over an $(n+1)$ dimensional manifold $\mathcal{M}$ with a Lagrangian density $L\left(F^{I}\right)$,

$$
\mathcal{A}\left[F^{I}\right]=\int_{\mathcal{M}} L\left(F^{I}\right)
$$


Under a variation of the fields the Lagrangian changes from $L$ to $L+\delta L$ with

$$
\delta L=E_{I} \wedge \delta F^{I}+d \theta,
$$

where

$$
E_{I}\left(F^{I}\right)=0
$$

are the equations of motion, $\theta\left(F^{I}, \delta F^{I}\right)$ is an $n$-form on $\mathcal{M}$.

Under a second variation of the fields we define $[3,5,7]$

$$
\omega\left(F^{I}, \delta_{1} F^{I}, \delta_{2} F^{I}\right):=\delta_{1} \theta_{2}-\delta_{2} \theta_{1},
$$

where $\theta_{1}=\theta\left(F^{I}, \delta_{1} F^{I}\right)$ and $\theta_{2}=\theta\left(F^{I}, \delta_{2} F^{I}\right)$. Demanding that $F^{I}$ are a solution of the equations of motion determines a point in the space of solutions, $\mathcal{S}$. We further demand that $\delta_{1} F^{I}$ and $\delta_{2} F^{I}$ are solutions of the linearized equations of motion (denoted $\delta_{1} S^{I}$ and $\delta_{2} S^{I}$ ) in the sense that

$E_{I}\left(S^{J}+\delta S^{J}\right) \approx E_{I}\left(S^{J}\right)+\delta E_{I}\left(S^{J}\right)=\delta E_{I}\left(S^{J}\right)=0$,

to first order in $\delta E^{J}$. Then $\theta$ is a 1 -form on $T^{*} \mathcal{S}$ and $\omega$ a 2-form (they are both $n$-forms on $T^{*} \mathcal{M}$ ).

In this context a field variation $\delta$ can be viewed as the exterior derivative on the space of solutions, with $\delta^{2}=0$. We shall adopt the convention that boldface symbols represent differential forms on $\mathcal{S}$ and write

$$
\omega=\delta \theta .
$$

From now on it will always be assumed that $S^{I}$ satisfy the equations of motion and all $\delta S^{I}$ satisfy the linearized equations of motion (7), so we are always dealing with differential forms on $\mathcal{S}$. For brevity we shall refer to such field configurations as "on shell". Thus Eq. (4) is

$$
\delta L=d \boldsymbol{\theta}
$$

on shell, and this then implies that

$$
0=\boldsymbol{\delta}^{2} L=\boldsymbol{\delta} d \boldsymbol{\theta}=d \boldsymbol{\delta} \boldsymbol{\theta}=d \boldsymbol{\omega},
$$

since the exterior derivative $d$ on $\mathcal{M}$ does not depend on the fields and $d \boldsymbol{\delta}=\boldsymbol{\delta} d$. Hence $\boldsymbol{\omega}$ is $d$-closed on shell.

Adding a total derivative to the action density does not change the equations of motion but can change $\omega$ by a total derivative. If

$$
L \rightarrow L^{\prime}=L+d \alpha
$$

then, again on shell,

$$
\boldsymbol{\delta} L^{\prime}=\boldsymbol{\delta} L+\boldsymbol{\delta} d \alpha=\boldsymbol{\delta} L+d \boldsymbol{\delta} \alpha=d(\boldsymbol{\theta}+\boldsymbol{\delta} \alpha)=d \boldsymbol{\theta}^{\prime}
$$

with

$$
\boldsymbol{\theta}^{\prime}=\boldsymbol{\theta}+\boldsymbol{\delta} \alpha+d \boldsymbol{\psi},
$$

where $\boldsymbol{\psi}$ is an $(n-2)$-form on $\mathcal{M}$ and a 1 -form on $\mathcal{S}$. Now

$$
\delta \boldsymbol{\theta}^{\prime}=\boldsymbol{\delta} \boldsymbol{\theta}+d \boldsymbol{\phi} \boldsymbol{\psi}
$$

so

$$
\omega^{\prime}=\omega+d \delta \psi
$$

Thus if $\Sigma$ is a hypersurface in $\mathcal{M}$ which is compact without boundary

$$
\boldsymbol{\Theta}^{\prime}:=\int_{\Sigma} \boldsymbol{\theta}^{\prime}=\int_{\Sigma} \boldsymbol{\theta}+\boldsymbol{\delta}\left(\int_{\Sigma} \alpha\right)=\boldsymbol{\Theta}+\boldsymbol{\delta}\left(\int_{\Sigma} \alpha\right)
$$

and

$$
\boldsymbol{\Omega}=\boldsymbol{\delta} \Theta=\int_{\Sigma} \omega=\int_{\Sigma} \omega^{\prime}=\delta \Theta^{\prime}
$$

is unchanged. If $\Sigma$ has a boundary $\partial \Sigma$ these expression are still valid provided $\int_{\partial \Sigma} \boldsymbol{\psi}=0$.

On the other hand the variation of the action

$$
\boldsymbol{\delta} \mathcal{A}=\int_{\mathcal{M}}\left(d \boldsymbol{\theta}+E_{I} \wedge \boldsymbol{\delta} S^{I}\right)=\int_{\mathcal{M}} d \boldsymbol{\theta}=\int_{\partial \mathcal{M}} \boldsymbol{\theta}
$$

on shell so, since $\boldsymbol{\delta}^{2}=0$,

$$
\boldsymbol{\delta}^{2} \mathcal{A}=\int_{\partial \mathcal{M}} \boldsymbol{\delta} \boldsymbol{\theta}=\int_{\partial \mathcal{M}} \boldsymbol{\omega}=0 .
$$

If $\partial \mathcal{M}$ consists of two spacelike hypersurfaces $\Sigma$ and $\Sigma^{\prime}$, connected by a timelike tube, ${ }^{2}$ on which the fields and $\boldsymbol{\theta}$ vanish then, with appropriate orientations for $\Sigma$ and $\Sigma^{\prime}$,

$$
\int_{\partial \mathcal{M}} \omega=\int_{\Sigma} \omega-\int_{\Sigma^{\prime}} \omega=0
$$

and

$$
\boldsymbol{\Omega}=\int_{\Sigma} \omega=\int_{\Sigma^{\prime}} \omega=\int_{\Sigma} \omega^{\prime}
$$

is independent of the spacelike hypersurface chosen and of any total derivatives added to the Lagrangian.

If $\Sigma$ is a Cauchy surface then $\boldsymbol{\Omega}$ is a presymplectic form on $\mathcal{S}$, in the sense of [3], and $\Theta$ is a presymplectic potential. They are not quite symplectic because we still need to factor out diffeomorphisms and restrict $\mathcal{S}$ to $\hat{\mathcal{S}}=\mathcal{S} / \mathcal{G}$.

\footnotetext{
${ }^{2}$ i.e., an $n$-dimensional space with one timelike direction and $(n-1)$ spacelike directions.
} 
The above discussion is summarized in the on shell commutative diagram below

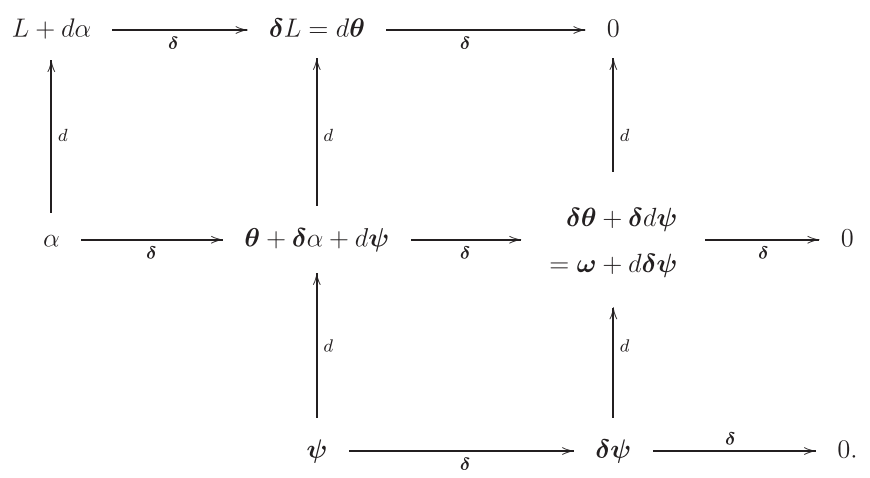

(15)

Mathematically the structure here is that of a doubly graded complex [19] and, as for any such complex, it can easily be reduced to a single complex. Let $W^{p, q}$ denote the space of $p$-forms on $\mathcal{S}$ and $q$-forms on $\mathcal{M}$. Then a singly graded complex is obtained by taking the space of constant total degree $r$,

$$
W^{r}=\bigoplus_{p+q=r} W^{p, q}
$$

and defining a differential operator

$$
\boldsymbol{D}=\boldsymbol{\delta}+(-1)^{p} d
$$

acting on $W^{r}$ and sending $W^{r} \rightarrow W^{r+1}$, with $\boldsymbol{D}^{2}=0$. Then $W^{0} \longrightarrow W^{1} \longrightarrow{ }_{D} \longrightarrow \underset{D}{\longrightarrow} W^{r} \longrightarrow D$

is a singly graded complex. In particular

$$
\begin{aligned}
\boldsymbol{D} & \{(L+d \alpha)+(\boldsymbol{\theta}+\boldsymbol{\delta} \alpha-d \boldsymbol{\psi})+\boldsymbol{\delta} \boldsymbol{\psi}\} \\
& =(\boldsymbol{\delta} L+\boldsymbol{\delta} d \alpha)+(\boldsymbol{\delta} \boldsymbol{\theta}-\boldsymbol{\delta} d \boldsymbol{\psi}-d \boldsymbol{\theta}-d \boldsymbol{\delta} \alpha)+d \boldsymbol{\delta} \boldsymbol{\psi} \\
& =\boldsymbol{\delta} \boldsymbol{\theta}=\boldsymbol{\omega} .
\end{aligned}
$$

Integrating the $n$-forms $\boldsymbol{\theta}$ and $\boldsymbol{\omega}$ over $\Sigma$ gives the following cohomology on the space of solutions:

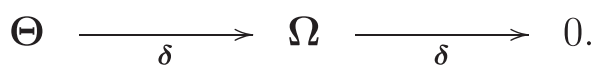

\section{DIFFEOMORPHISMS AND KILLING SYMMETRIES}

\section{A. General diffeomorphisms}

We would like to understand how diffeomorphisms fit into this picture. Diffeomorphisms are generated by an infinitesimal vector field $\epsilon \vec{X}$ with $\epsilon$ an infinitesimally small constant. $\vec{X}$ generates a variation of the fields

$$
\boldsymbol{\delta} F^{I}=\epsilon \mathcal{L}_{\vec{X}} F^{I}=\epsilon d i_{\vec{X}} F^{I}+\epsilon i_{\vec{X}} d F^{I} .
$$

One might expect that, since $\vec{X}$ is a fixed vector field independent of the fields $F^{I}$,

$$
\boldsymbol{\delta} \mathcal{L}_{\vec{X}}=\mathcal{L}_{\vec{X}} \boldsymbol{\delta} \quad \text { and } \quad \delta i_{\vec{X}}=i_{\vec{X}} \boldsymbol{\delta}
$$

but we should remember that we are interpreting $\boldsymbol{\epsilon} \mathcal{L}_{\vec{X}} F^{I}$ as a 1 -form on $\mathcal{S}$. It is convenient to promote $\epsilon$ to be a constant Grassmann parameter $\boldsymbol{\epsilon}$, where $\boldsymbol{\epsilon}$ is a 1 -form on $\mathcal{S}$ which anticommutes with $\boldsymbol{\delta}$, and write

$$
\boldsymbol{\delta}_{\vec{X}}=\boldsymbol{\epsilon} \mathcal{L}_{\vec{X}}
$$

with

$$
\boldsymbol{\delta}_{\vec{X}} \boldsymbol{\delta}=-\delta \boldsymbol{\delta}_{\vec{X}}
$$

This maintains the condition $d \boldsymbol{\delta}=\boldsymbol{\delta} d$, since $i_{\vec{X}} \boldsymbol{\delta}=\boldsymbol{\delta} i_{\vec{X}}$.

It is then natural to decompose the exterior derivative $\delta$ on $\mathcal{S}$ into a genuine physical variation of the fields $\hat{\boldsymbol{\delta}}$ and a variation arising from diffeomorphisms,

$$
\boldsymbol{\delta}=\hat{\boldsymbol{\delta}}+\boldsymbol{\delta}_{\vec{X}}=\hat{\boldsymbol{\delta}}+\boldsymbol{\epsilon} \mathcal{L}_{\vec{X}} .
$$

If the solution depends on a set of parameters (moduli) then the variation $\hat{\delta}$ can be induced by varying the parametersit is an exterior derivative on the moduli space. This decomposition is unique, if there were different decompositions

$$
\boldsymbol{\delta}=\hat{\boldsymbol{\delta}}+\boldsymbol{\delta}_{\vec{X}}=\hat{\boldsymbol{\delta}}^{\prime}+\boldsymbol{\delta}_{\vec{X}^{\prime}}
$$

then

$$
\hat{\boldsymbol{\delta}}-\hat{\boldsymbol{\delta}}^{\prime}=\boldsymbol{\delta}_{\vec{X}}-\boldsymbol{\delta}_{\vec{X}^{\prime}}
$$

and both sides must vanish since the left-hand side is a genuine physical variation of the fields and the right-hand side is a diffeomorphism.

There is a similar decomposition of forms, any 1-form $\boldsymbol{\eta}$ on $\mathcal{S}$ (and $q$-form on $\mathcal{M}$ ) can be decomposed as

$$
\boldsymbol{\eta}=\hat{\boldsymbol{\eta}}+\boldsymbol{\eta}_{\vec{X}}=\hat{\boldsymbol{\eta}}+\boldsymbol{\epsilon} \eta(\vec{X}),
$$

where $\boldsymbol{\eta}_{\vec{X}}=\boldsymbol{\epsilon} \eta(\vec{X})$ with $\eta(\vec{X})$ a $q$-form on $\mathcal{M}$ and a function on $\mathcal{S}$. In particular

$$
\boldsymbol{\theta}=\hat{\boldsymbol{\theta}}+\boldsymbol{\theta}_{\vec{X}}=\hat{\boldsymbol{\theta}}+\boldsymbol{\epsilon} \theta(\vec{X}),
$$

where $\theta(\vec{X})$ is an $n$-form on $\mathcal{M}$. Then 


$$
d \boldsymbol{\theta}=\boldsymbol{\delta} L \Rightarrow\left\{\begin{array}{l}
d \hat{\boldsymbol{\theta}}=\hat{\boldsymbol{\delta}} L, \\
d \theta(\vec{X})=d i_{\vec{X}} L .
\end{array}\right.
$$

The Lagrangian $L$ itself is not diffeomorphism invariant but rather, under a variation which is a diffeomorphism generated by a vector field $\vec{X}$, we have

$$
\boldsymbol{\delta}_{\vec{X}} L=\boldsymbol{\epsilon} d i_{\vec{X}} L=d \boldsymbol{\theta}_{\vec{X}}
$$

on shell, and all we can deduce from this is that

$$
\boldsymbol{\theta}_{\vec{X}}=\boldsymbol{\epsilon} i_{\vec{X}} L+\boldsymbol{J}_{\vec{X}}=\boldsymbol{\epsilon}\left(i_{\vec{X}} L+J(\vec{X})\right)
$$

with

$$
J(\vec{X}):=\theta(\vec{X})-i_{\vec{X}} L,
$$

and $d J(\vec{X})=0 . J(\vec{X})$ is referred to as the Noether current in [7].

Now $J(\vec{X})$ is closed, and we shall assume that it is $d$-exact on shell. We shall see below, in Eq. (29), that if this is not the case there is an obstruction to defining a genuine symplectic structure on $\hat{\mathcal{S}}$. So we assume that

$$
J(\vec{X})=d Q(\vec{X})
$$

on shell and then

$$
\boldsymbol{\theta}_{\vec{X}}=\boldsymbol{\epsilon} i_{\vec{X}} L+d \boldsymbol{Q}_{\vec{X}}=\boldsymbol{\epsilon}\left(i_{\vec{X}} L+d Q(\vec{X})\right) .
$$

In fact it is argued in [6] that, subject to some mild conditions, $d J(\vec{X})=0$ implies that $J(\vec{X})$ is $d$-exact but there are some subtleties in the argument, ${ }^{3}$ so we shall simply assume that $J(\vec{X})$ is $d$-exact.

This then has important consequences for the presymplectic density $\boldsymbol{\omega}$. We have

$$
\begin{aligned}
\boldsymbol{\omega} & =\boldsymbol{\delta} \boldsymbol{\theta}=\left(\hat{\boldsymbol{\delta}}+\boldsymbol{\delta}_{\vec{X}}\right)\left(\hat{\boldsymbol{\theta}}+\boldsymbol{\theta}_{\vec{X}}\right) \\
& =\hat{\boldsymbol{\delta}} \hat{\boldsymbol{\theta}}+\boldsymbol{\delta}_{\vec{X}} \hat{\boldsymbol{\theta}}+\hat{\boldsymbol{\delta}} \boldsymbol{\theta}_{\vec{X}}+\boldsymbol{\delta}_{\vec{X}} \boldsymbol{\theta}_{\vec{X}} \\
& =\hat{\boldsymbol{\omega}}+\boldsymbol{\delta}_{\vec{X}} \hat{\boldsymbol{\theta}}+\hat{\boldsymbol{\delta}} \boldsymbol{\theta}_{\vec{X}},
\end{aligned}
$$

where $\hat{\boldsymbol{\omega}}=\hat{\boldsymbol{\delta}} \hat{\boldsymbol{\theta}}$ and $\boldsymbol{\delta}_{\vec{X}} \boldsymbol{\theta}_{\vec{X}}$ vanishes because $\boldsymbol{\epsilon}^{2}=0$. Now

\footnotetext{
${ }^{3}$ For example it does not apply to the presymplectic density $\omega$ which is also $d$-closed (10). One of the conditions in [6] for a closed form which depends on some dynamical fields to be exact is that it must vanish when the fields vanish. As long as $\boldsymbol{\omega}$ can be put in Darboux form (e.g., see [5] for the case of Einstein gravity and [12] for Einstein gravity with a cosmological constant) it does not vanish when the fields vanish, because the components are constant in Darboux coordinates.
}

$$
\begin{aligned}
\boldsymbol{\delta}_{\vec{X}} \hat{\boldsymbol{\theta}}+\hat{\boldsymbol{\delta}} \boldsymbol{\theta}_{\vec{X}} & =\boldsymbol{\epsilon} \mathcal{L}_{\vec{X}} \hat{\boldsymbol{\theta}}+\hat{\boldsymbol{\delta}}\left(\boldsymbol{\epsilon} i_{\vec{X}} L+\boldsymbol{\epsilon} J(\vec{X})\right) \quad \text { using } \\
& =\boldsymbol{\epsilon}\left(\left(d i_{\vec{X}} \hat{\boldsymbol{\theta}}+i_{\vec{X}} d \hat{\boldsymbol{\theta}}\right)-i_{\vec{X}} \hat{\boldsymbol{\delta}} L-\hat{\boldsymbol{\delta}} d Q(\vec{X})\right) \\
& =\boldsymbol{\epsilon}\left(d i_{\vec{X}} \hat{\boldsymbol{\theta}}-\hat{\boldsymbol{\delta}} d Q(\vec{X})\right) \quad \text { using } \\
& :=\boldsymbol{\epsilon} d \phi(\vec{X})
\end{aligned}
$$

where

$$
\boldsymbol{\phi}(\vec{X})=i_{\vec{X}} \hat{\boldsymbol{\theta}}-\hat{\boldsymbol{\delta}} Q(\vec{X}) \bmod d
$$

Since

$$
\boldsymbol{\delta}_{\vec{X}} \hat{\boldsymbol{\theta}}+\hat{\boldsymbol{\delta}} \boldsymbol{\theta}_{\vec{X}}=\boldsymbol{\omega}\left(\boldsymbol{\delta}_{\vec{X}} F^{I}, \hat{\boldsymbol{\delta}} F^{I}\right)
$$

this relates to the Hamiltonian flow [7] -if there is a Hamiltonian $\mathcal{H}[\vec{X}]$ on phase space that generates the evolution corresponding to the flow arising from $\vec{X}$ (not necessarily timelike) then

$$
\boldsymbol{\omega}\left(\boldsymbol{\delta}_{\vec{X}} F^{I}, \hat{\boldsymbol{\delta}} F^{I}\right)=-\hat{\boldsymbol{\delta}} \boldsymbol{h}_{\vec{X}}=\boldsymbol{\epsilon} \hat{\boldsymbol{\delta}} h(\vec{X})
$$

with

$$
\hat{\boldsymbol{\delta}} h(\vec{X})=d \phi(\vec{X})=d\left\{i_{\vec{X}} \hat{\boldsymbol{\theta}}-\hat{\boldsymbol{\delta}} Q(\vec{X})\right\}
$$

the variation of the corresponding Hamiltonian density. In that case we can define a Hamiltonian $\mathcal{H}[\vec{X}]$ which satisfies

$$
\hat{\boldsymbol{\delta}} \mathcal{H}[\vec{X}]=\int_{\Sigma} \hat{\boldsymbol{\delta}} h(\vec{X})=\int_{\partial \Sigma}\left(i_{\vec{X}} \hat{\boldsymbol{\theta}}-\hat{\boldsymbol{\delta}} Q(X)\right) .
$$

If $\vec{X}$ does not vanish on the boundary and

$$
\int_{\partial \Sigma} i_{\vec{X}} \hat{\boldsymbol{\theta}} \neq 0
$$

then a Hamiltonian corresponding to the flow generated by $\vec{X}$ will only exist if $\int_{\partial \Sigma} i_{\vec{X}} \hat{\boldsymbol{\theta}}$ is $\boldsymbol{\delta}$-exact. The existence of such a Hamiltonian requires this integrability condition which is decided by the specific theory in question, [7].

We can see that $h(\vec{X})$ and $J(\vec{X})$ are well-defined in cohomology. Under the change $L \rightarrow L+d \alpha$ and $\boldsymbol{\theta} \rightarrow \boldsymbol{\theta}+$ $\boldsymbol{\delta} \alpha+d \boldsymbol{\psi}$,

$$
\begin{aligned}
\boldsymbol{J}_{\vec{X}} & =\boldsymbol{\theta}_{\vec{X}}-\boldsymbol{\epsilon} i_{\vec{X}} L \rightarrow \boldsymbol{J}_{\vec{X}}+d\left(\boldsymbol{\epsilon} i_{\vec{X}} \alpha+\boldsymbol{\psi}_{\vec{X}}\right) \\
& =\boldsymbol{\epsilon}\left\{J(\vec{X})+d\left(i_{\vec{X}} \alpha+\psi(\vec{X})\right)\right\}
\end{aligned}
$$

is unchanged if we choose $\psi(\vec{X})=-i_{\vec{X}} \alpha \bmod d$. This together with

$$
\hat{\boldsymbol{\theta}} \rightarrow \hat{\boldsymbol{\theta}}+\hat{\boldsymbol{\delta}} \alpha+d \hat{\boldsymbol{\psi}}
$$

then shows that 


$$
\begin{aligned}
\boldsymbol{\epsilon} \hat{\boldsymbol{\delta}} h(\vec{X}) & \rightarrow \boldsymbol{\epsilon} \hat{\boldsymbol{\delta}} h(\vec{X})-\hat{\boldsymbol{\delta}} \boldsymbol{\epsilon} d\left(i_{\vec{X}} \alpha+\psi(\vec{X})\right)-\boldsymbol{\epsilon} d i_{\vec{X}}(\hat{\boldsymbol{\delta}} \alpha+d \hat{\boldsymbol{\psi}}) \\
& =\hat{\boldsymbol{\delta}} \boldsymbol{h}_{\vec{X}}+\boldsymbol{\epsilon}\left\{\hat{\boldsymbol{\delta}} d \psi(\vec{X})-d i_{\vec{X}} d \hat{\boldsymbol{\psi}}\right\}
\end{aligned}
$$

is unchanged provided we choose $\hat{\boldsymbol{\psi}}$ such that

$$
i_{\vec{X}} d \hat{\boldsymbol{\psi}}=\hat{\boldsymbol{\delta}} \psi(\vec{X})=-i_{\vec{X}} \hat{\boldsymbol{\delta}} \alpha \bmod d .
$$

In any case, whether or not a Hamiltonian exists, we have, assuming $J=d Q$ on shell,

$$
\boldsymbol{\delta}_{\vec{X}} \hat{\boldsymbol{\theta}}+\hat{\boldsymbol{\delta}} \boldsymbol{\theta}_{\vec{X}}=d \phi_{\vec{X}}
$$

with

$$
\phi_{\vec{X}}:=\boldsymbol{\epsilon} i_{\vec{X}} \hat{\boldsymbol{\theta}}+\hat{\boldsymbol{\delta}} \boldsymbol{Q}_{\vec{X}} \bmod d
$$

and

$$
\boldsymbol{\epsilon} i_{\vec{X}} \hat{\boldsymbol{\theta}}+\hat{\boldsymbol{\delta}} \boldsymbol{Q}_{\vec{X}}=\boldsymbol{\epsilon}\left(i_{\vec{X}} \hat{\boldsymbol{\theta}}-\hat{\boldsymbol{\delta}} Q(\vec{X})\right) .
$$

This results in the important conclusion that, for a diffeomorphism $\vec{X}$,

$$
\boldsymbol{\omega}=\hat{\boldsymbol{\omega}}+d \phi_{\vec{X}},
$$

and this guarantees that

$$
\hat{\boldsymbol{\omega}}=\hat{\boldsymbol{\delta}} \hat{\boldsymbol{\theta}}+\hat{\boldsymbol{\delta}} \phi_{\vec{X}}
$$

is a bona fide symplectic density on $T^{*} \hat{\mathcal{S}}$ when $\Sigma$ is compact without boundary, because it pulls back to $\omega \bmod d$ under the projection from $\mathcal{S}$ to $\hat{\mathcal{S}}$ as demanded in [3]. If $\Sigma$ is compact without boundary it follows that $\hat{\mathbf{\Omega}}$ on $\hat{\mathcal{S}}$ pulls back to the presymplectic form $\boldsymbol{\Omega}$ on $T^{*} \mathcal{S}$. If $\Sigma$ has a boundary $\partial \Sigma$ then we must restrict the diffeomorphisms to allow only those for which the vector fields generating them fall off fast enough at the boundary so that surface terms vanish.

The essence of the above formulas is summarized in the following on shell diagram:

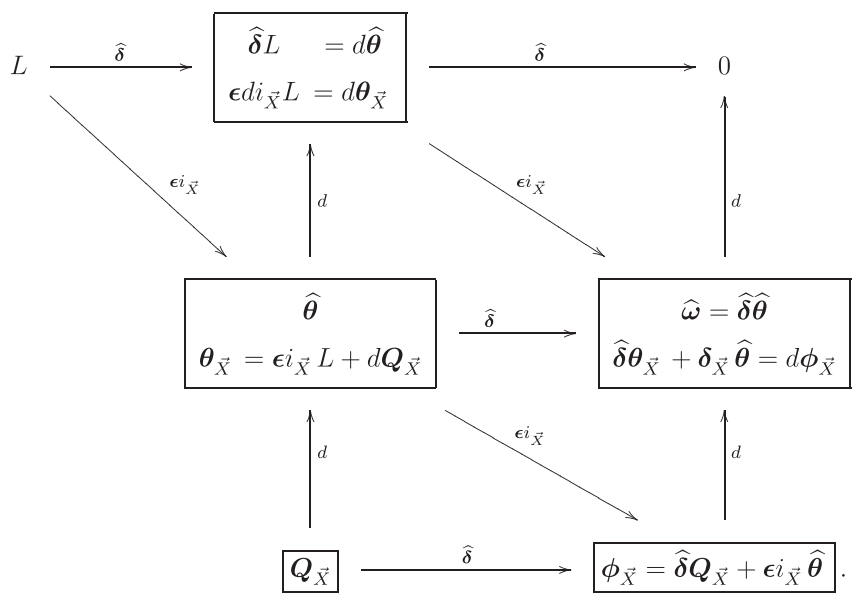

Integrating the upper member of the middle row over $\Sigma$ gives

$$
\hat{\Theta}=\int_{\Sigma} \hat{\boldsymbol{\theta}} \stackrel{\hat{\boldsymbol{\delta}}}{\rightarrow} \hat{\Omega}=\int_{\Sigma} \hat{\boldsymbol{\omega}}
$$

We have here a double complex [19] whose general structure is

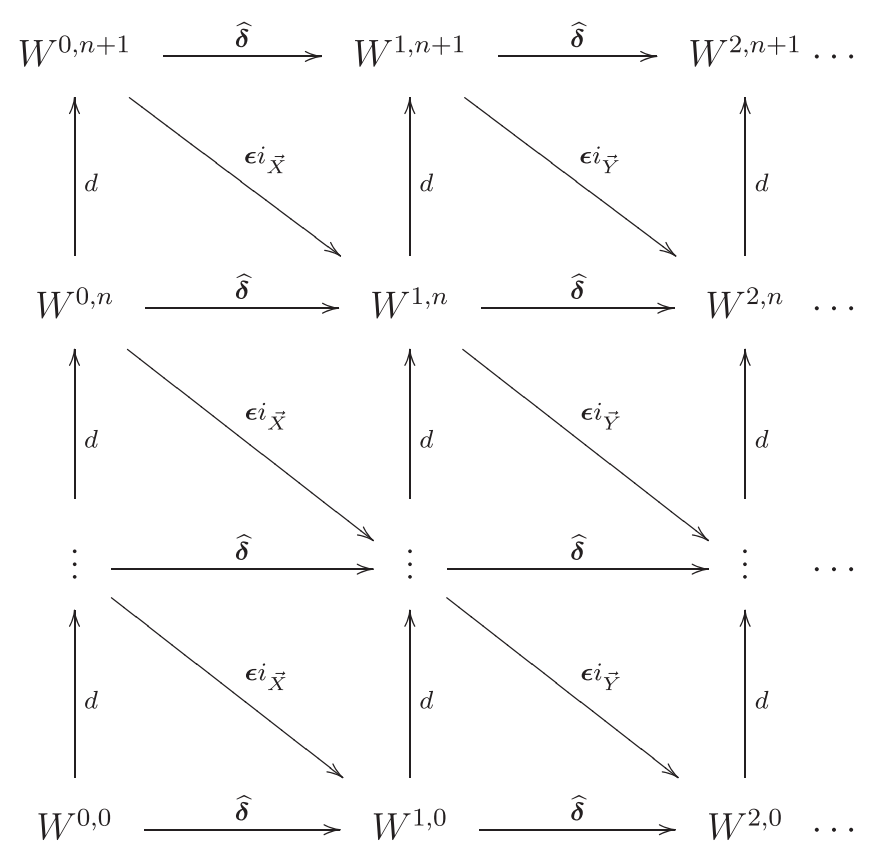

In principle subsequent diffeomorphisms could be generated by different vector fields $\vec{X}$ and $\vec{Y}$, though the analysis above assumed $\vec{X}=\vec{Y}$ in order to understand how a single diffeomorphism affected $\boldsymbol{\omega}$ in (29).

Note that $\boldsymbol{\epsilon} i_{\vec{X}}$ preserves the total degree $p+q$ of the forms on $W^{r}$.

\section{B. Killing symmetries and conserved charges}

The formalism really comes into its own for discussing symmetries. If the classical action has symmetries that are broken at the quantum level there are anomalies and the double complex sketched in (30) is the natural framework for analyzing the Stora-Zumino descent equations. These can include gravitational anomalies associated with local Lorentz invariance and diffeomorphism anomalies, which are equivalent [2], but we shall not analyze anomalies here.

Consider (27) when $\vec{X}=\vec{K}$

$$
\boldsymbol{\delta}_{\vec{K}} \hat{\boldsymbol{\theta}}+\hat{\boldsymbol{\delta}} \boldsymbol{\theta}_{\vec{K}}=\hat{\boldsymbol{\delta}} \boldsymbol{\theta}_{\vec{K}}=d \phi_{\vec{K}}
$$

and, for $\vec{K}$ Killing,

$$
\boldsymbol{\epsilon} \mathcal{L}_{\vec{K}} L=\boldsymbol{\epsilon} d i_{\vec{K}} L=d \boldsymbol{\theta}_{\vec{K}}=0
$$


so $\boldsymbol{\theta}_{\vec{K}}$ is $d$-closed. In fact since $\boldsymbol{\theta}_{\vec{K}}$ is linear in $\mathcal{L}_{\vec{K}} F^{I}$ and $\mathcal{L}_{\vec{K}} \partial^{k} F^{I}$ we expect that $\boldsymbol{\theta}_{\vec{K}}=0$ when $\vec{K}$ is Killing. ${ }^{4}$ For the same reason $\boldsymbol{\delta}_{\vec{K}} \hat{\boldsymbol{\theta}}=0$ and

$$
d \phi_{\vec{K}}=0
$$

with

$$
\boldsymbol{\phi}_{\vec{K}}=\boldsymbol{\epsilon} i_{\vec{K}} \hat{\boldsymbol{\theta}}+\hat{\boldsymbol{\delta}} Q_{\vec{K}}=\boldsymbol{\epsilon}\left(i_{\vec{K}} \hat{\boldsymbol{\theta}}-\hat{\boldsymbol{\delta}} Q(\vec{K})\right) .
$$

Two important conclusions immediately follow:

(i) From (29) and (32)

$$
\omega=\hat{\omega}
$$

for a Killing symmetry.

(ii) If we can foliate $\Sigma$ into hypersurfaces $\sigma_{r}$ (e.g., $r$ could be a radial coordinate) then we can integrate over a piece of $\Sigma$ which is a thick shell $\Sigma_{\left[r, r^{\prime}\right]}$ between $r$ and $r^{\prime}$ and

$$
\int_{\Sigma_{\left[r, r^{\prime}\right]}} d \phi_{\vec{K}}=\int_{\sigma_{r^{\prime}}} \phi_{\vec{K}}-\int_{\sigma_{r}} \phi_{\vec{K}}=0
$$

implies that

$$
\boldsymbol{\Phi}[\vec{K}]:=\int_{\sigma_{r}} \phi(\vec{K})=\int_{\sigma_{r}}\left(i_{\vec{K}} \hat{\boldsymbol{\theta}}-\hat{\boldsymbol{\delta}} Q(\vec{K})\right),
$$

a 1 -form on $T^{*} \mathcal{S}$, is independent of $r$. For example $\sigma_{r}$ might be an $(n-1)$ sphere, and it can be convenient to evaluate $\boldsymbol{\Phi}$ at $r \rightarrow \infty$ but the formalism here shows that this is not essential. Any value of $r$ can be used in principle, though in practice it is usually easier to do the integrals at $r \rightarrow \infty$. It is not even necessary to use a round sphere.

Recalling the discussion of the Hamiltonian there may be an obstruction to obtaining a genuine charge from $\boldsymbol{\Phi}[\vec{K}]$, it is a 1 -form on $T^{*} \mathcal{S}$ and does not yet yield a charge. A genuine charge emerges from this construction only if $i_{\vec{K}} \hat{\boldsymbol{\theta}}$ is $\hat{\boldsymbol{\delta}}$-exact. If this is this case, and only if this is the case, we can write

$$
i_{\vec{K}} \hat{\boldsymbol{\theta}}=\hat{\boldsymbol{\delta}} \mu(\vec{K})
$$

and define

$$
\rho(\vec{K})=\mu(\vec{K})-Q(\vec{K})
$$

\footnotetext{
${ }^{4}$ For gauge theories this might not be strictly true. For electromagnetism, e.g., $L=-\frac{1}{2} F \wedge * F$ and $\boldsymbol{\theta}_{\vec{K}}=-\boldsymbol{\epsilon}\left(\mathcal{L}_{\vec{K}} A\right) \wedge * F$. While $\mathcal{L}_{\vec{K}} * F=0$ by assumption $\mathcal{L}_{\vec{K}} A$ might not be. But on shell $\boldsymbol{\theta}_{\vec{K}}=-\boldsymbol{\epsilon}\left(i_{\vec{K}} F\right) \wedge * F \bmod d$ and so is invariant $\bmod d$ under a gauge transformation.
}

with $\rho(\vec{K})$ an $(n-1)$-form on $\mathcal{M}$ satisfying

$$
\hat{\boldsymbol{\delta}} \rho(\vec{K})=\boldsymbol{\phi}(\vec{K}), \quad \hat{\boldsymbol{\delta}} h(\vec{K})=\hat{\boldsymbol{\delta}} d \rho(\vec{K}) .
$$

When $\vec{K}$ is Killing $d \phi(\vec{K})=0$ and we then have an invariant

$$
\hat{\boldsymbol{\delta}} \mathcal{H}[\vec{K}]=\int_{\partial \Sigma} \hat{\boldsymbol{\delta}} \rho(\vec{K})=0 .
$$

Again if $\partial \Sigma=\sigma_{r} \cup \sigma_{r^{\prime}}$

$$
\hat{\boldsymbol{\delta}} \mathcal{Q}:=\int_{\sigma_{r}} \hat{\boldsymbol{\delta}} \rho(\vec{K})=\int_{\sigma_{r^{\prime}}} \hat{\boldsymbol{\delta}} \rho(\vec{K})
$$

is independent of which copy of $\sigma$ it is evaluated on. We can associate a Noether charge ${ }^{5}$

$$
\mathcal{Q}[\vec{K}]=\int_{\sigma} \rho(\vec{K})
$$

with the symmetry $\vec{K}$, for which

$$
\hat{\boldsymbol{\delta}} \mathcal{Q}[\vec{K}]=\boldsymbol{\Phi}[\vec{K}] .
$$

For example for a stationary space-time, with Killing vector $\vec{K}=\frac{\partial}{\partial t}, \mathcal{Q}[\vec{K}]$ is a mass while for an axially symmetric space-time, with Killing vector $\vec{K}=\frac{\partial}{\partial \varphi}, \mathcal{Q}[\vec{K}]$ is the angular momentum associated with the space-time.

In summary, given a solution of the equations of motion with Killing vector $\vec{K}$, we define the $(n-1)$-form $Q(\vec{K})$ $\bmod d$ by

$$
d Q(\vec{K})=\theta(\vec{K})-i_{\vec{K}} L .
$$

Then, if and only if $i_{\vec{K}} \boldsymbol{\theta}=\hat{\boldsymbol{\delta}} \mu(\vec{K})$ is $\boldsymbol{\delta}$-exact, we have

$$
\hat{\boldsymbol{\delta}} \mathcal{Q}[\vec{K}]=\hat{\boldsymbol{\delta}} \int_{\sigma_{r}} \rho(\vec{K})=\int_{\sigma_{r}}\left(i_{\vec{K}} \hat{\boldsymbol{\theta}}-\hat{\boldsymbol{\delta}} Q(\vec{K})\right) .
$$

In analogy with (2) we define a 2-form to be the Hodge dual of $\rho(\vec{K})$,

$$
\rho(\vec{K})=* \mathcal{J}(\vec{K})
$$

and

$$
\mathcal{Q}[\vec{K}]=\int_{\sigma} * \mathcal{J}(\vec{K})
$$

with $d * \mathcal{J}=0$ on shell. For a space-time symmetry generated by a Killing vector $\vec{K}$ the analogue of a Noether current is a Noether 2-form.

\footnotetext{
${ }^{5}$ In general this is not the same as Wald's Noether charge associated with the entropy in [7].
} 
We have the sequence

$$
\mathcal{Q} \underset{\widehat{\delta}}{\longrightarrow} \Phi \underset{\widehat{\delta}}{\longrightarrow} 0 \text {. }
$$

\section{EXAMPLE: EINSTEIN GRAVITY AND THE SCHWARZSCHILD GEOMETRY}

As an example of these ideas example consider Einstein gravity in four dimensions. The Lagrangian is

$$
L=\frac{1}{16 \pi} R_{a b} \wedge * e^{a b},
$$

where $e^{a}, a=0,1,2,3$ are orthonormal 1-forms,

$$
R_{a b}=d \omega_{a b}+\omega_{a c} \wedge \omega_{b}^{c},
$$

are the curvature 2-forms for the associated connection 1 -forms $\omega_{a b}$ and $*$ is the Hodge star. The connection 1 -forms are determined by the torsion free condition

$$
D e^{a}=d e^{a}+\omega_{b}^{a} \wedge e^{b}=0
$$

and orthonormal indices are lowered with $\eta_{a b}=$ $\operatorname{diag}(-1,0,0,0)$.

Under a variation of the 1 -forms, $e^{a} \rightarrow e^{a}+\delta e^{a}$,

$$
\delta R_{a b}=D\left(\delta \omega_{a b}\right)
$$

and

$$
\begin{aligned}
\delta L & =\frac{1}{16 \pi}\left\{\delta e^{c} \wedge E_{c}+D\left(\delta \omega_{a b}\right) \wedge * e^{a b}\right\} \\
& =\frac{1}{16 \pi}\left\{\left(\delta e^{c} \wedge E_{c}+d\left(\left(\delta \omega_{a b}\right) \wedge * e^{a b}\right)\right\},\right.
\end{aligned}
$$

where Einstein's equations are

$$
E^{c}=R_{a b} \wedge * e^{a b c}=0 .
$$

From this we get ${ }^{6}$

$$
\theta\left(\delta e^{a}\right)=\left(\delta \omega_{a b}\right) \wedge * e^{a b}
$$

\footnotetext{
${ }^{6}$ There is a subtlety here, not all $\delta e^{a}$ correspond to genuine variations in the metric some are just local tangent space rotations. Expanding $\delta e^{a}=\Delta^{a}{ }_{b} e^{b}$ only the symmetric part of $\Delta_{a b}$ can give genuine metric variations (and some of these are just diffeomorphisms), the antisymmetric part of $\Delta_{a b}$ is a tangent space rotation (Lorentz transformation). We ignore this problem here and just choose a gauge in which $\Delta_{a b}$ is symmetric, but this is not necessary. This relates to the fact that (9) only defines $\boldsymbol{\theta}$ mod $d$, in general the antisymmetric part of $\Delta_{a b}$ can be eliminated by adding a $d$-exact form to $\boldsymbol{\theta}$. Full details are given in [12].
}

For a diffeomorphism, $\delta e^{a}=\mathcal{L}_{\vec{X}} e^{a}$,

$$
\theta\left(\mathcal{L}_{\vec{X}} e^{a}\right)=\frac{1}{16 \pi}\left(\mathcal{L}_{\vec{X}} \omega_{a b}\right) \wedge * e^{a b}=-\frac{1}{16 \pi} d * d X
$$

on shell. Also

$$
\begin{aligned}
\mathcal{L}_{\vec{X}} \theta\left(\delta e^{a}\right) & =\frac{1}{16 \pi}\left\{d i_{\vec{X}}\left(\delta \omega_{a b} \wedge * e^{a b}\right)+i_{\vec{X}} d\left(\delta \omega_{a b} \wedge * e^{a b}\right)\right\} \\
& =\frac{1}{16 \pi}\left\{d i_{\vec{X}}\left(\delta \omega_{a b} \wedge * e^{a b}\right)+i_{\vec{X}}\left(D\left(\delta \omega_{a b}\right) \wedge * e^{a b}\right)\right\} \\
& =\frac{1}{16 \pi}\left\{d i_{\vec{X}}\left(\delta \omega_{a b} \wedge * e^{a b}\right)+i_{\vec{X}}\left(\left(\delta R_{a b}\right) \wedge * e^{a b}\right)\right\}
\end{aligned}
$$

By assumption $\delta e^{a}$ satisfies the linearized equations of motion so

$$
\delta R_{a b} \wedge * e^{a b c}=0 \Rightarrow \delta R_{a b} \wedge * e^{a b}=0 .
$$

Combining (38) and (39) we conclude that the presymplectic density satisfies

$$
\begin{aligned}
\omega\left(\mathcal{L}_{\vec{X}} e^{a}, \delta e^{a},\right)= & \mathcal{L}_{\vec{X}} \theta\left(\delta e^{a}\right)-\delta \theta\left(\mathcal{L}_{\vec{X}} e^{a}\right) \\
= & \left(\delta \omega_{a b}\right) \wedge\left(\mathcal{L}_{\vec{X}} * e^{a b}\right) \\
& -\left(\mathcal{L}_{\vec{X}} \omega_{a b}\right) \wedge\left(\delta * e^{a b}\right) \\
= & \frac{1}{16 \pi} d\left\{i_{\vec{X}}\left(\delta \omega_{a b} \wedge * e^{a b}\right)+\delta(* d X)\right\},
\end{aligned}
$$

and we have obtained

$$
\phi(\vec{X})=\frac{1}{16 \pi}\left\{i_{\vec{X}}\left(\delta \omega_{a b} \wedge * e^{a b}\right)+\delta(* d X)\right\}
$$

for Einstein gravity. When $\vec{X}=\vec{K}$ is Killing both $\theta\left(\mathcal{L}_{\vec{K}} e^{a}\right)$ and the symplectic form $\omega\left(\delta e^{a}, \mathcal{L}_{\vec{K}} e^{a}\right)$ vanish and $* d K$ is the Komar 2-form.

\section{A. Derivation of Noether mass for the Schwarzschild solution}

To illustrate the above ideas we consider the Schwarzschild metric

$$
\begin{aligned}
d s^{2}= & -\left(1-\frac{2 m}{r}\right) d t^{2}+\left(1-\frac{2 m}{r}\right)^{-1} d r^{2} \\
& +r^{2}\left(d \vartheta^{2}+\sin ^{2} \vartheta d \varphi^{2}\right) .
\end{aligned}
$$

We choose orthonormal 1-forms 


$$
\begin{array}{ll}
e^{0}=\sqrt{1-\frac{2 m}{r}} d t, \quad e^{2}=r d \vartheta, \\
e^{1}=\frac{d r}{\sqrt{1-\frac{2 m}{r}}}, \quad e^{2}=r \sin \vartheta d \varphi,
\end{array}
$$

giving connection 1-forms

$$
\begin{aligned}
& \omega_{01}=-\frac{m}{r^{2}} d t, \quad \omega_{02}=\omega_{03}=0, \\
& \omega_{12}=-\sqrt{1-\frac{2 m}{r}} d \vartheta, \\
& \omega_{13}=-\sqrt{1-\frac{2 m}{r}} \sin \vartheta d \varphi, \omega_{23}=-\cos \vartheta d \varphi .
\end{aligned}
$$

We shall calculate the mass associated with the Killing vector

$$
\vec{K}=\frac{\partial}{\partial t}
$$

and its metric dual 1-form

$$
K=-\left(1-\frac{2 m}{r}\right) d t
$$

[with signature $(-,+,+,+)]$. We have

$$
d K=\frac{2 m}{r^{2}} e^{01}
$$

and

$$
* d K=\frac{2 m}{r^{2}} e^{23}=2 m \sin \vartheta d \vartheta \wedge d \varphi
$$

(we use conventions with $1=e^{0123}$ and $* e^{01}=e^{23}$ ). Note that $d * d K=0$ so $\theta(\vec{K})=0$.

Now suppose the metric variation $\delta e^{a}$ is induced by varying the parameter $m$. For this variation

$$
\delta e^{0}=-\frac{\delta m}{r-2 m} e^{0}, \quad \delta e^{1}=\frac{\delta m}{r-2 m} e^{1}, \quad \delta e^{2}=\delta e^{3}=0,
$$

with $\delta m$ constant, while (42) gives

$$
\begin{aligned}
& \delta \omega_{01}=-\frac{\delta m}{r^{2}} \frac{e^{0}}{\sqrt{1-\frac{2 m}{r}}}, \\
& \delta \omega_{12}=\frac{\delta m}{r^{2}} \frac{e^{2}}{\sqrt{1-\frac{2 m}{r}}}, \\
& \delta \omega_{13}=\frac{\delta m}{r^{2}} \frac{e^{3}}{\sqrt{1-\frac{2 m}{r}}} .
\end{aligned}
$$

The variations (45) produce

$$
i_{\vec{K}}\left(\delta \omega_{a b} \wedge * e^{a b}\right)=\frac{2 \delta m}{r^{2}} e^{23} .
$$

Also from (44)

$$
\delta(* d K)=\frac{2 \delta m}{r^{2}} e^{23},
$$

and these combine in (41) to give

$$
\phi(\vec{K})=\frac{1}{4 \pi} \frac{\delta m}{r^{2}} e^{23}=\frac{\delta m}{4 \pi} \sin \vartheta d \vartheta d \varphi .
$$

Now we choose constant time slices $\Sigma$ with $2 m<r<\infty$, $0 \leq \vartheta \leq \pi$ and $0 \leq \varphi<2 \pi$. $\sigma_{r}$ are 2 -spheres of radius $r$, and

$$
\delta \mathcal{Q}(\vec{K})=\int_{\sigma_{r}} \phi(\vec{K})=\frac{\delta m}{4 \pi} \int_{0}^{\pi} \int_{0}^{2 \pi} \sin \vartheta d \vartheta d \varphi=\delta m
$$

so

$$
\mathcal{Q}=\frac{1}{4 \pi} \int_{S^{2}} m \sin \vartheta d \vartheta d \varphi,=m
$$

The parameter $m$ in the Schwarzschild metric is indeed the mass, as expected.

Note that the final answer is independent of $r$, it is not necessary to take $r \rightarrow \infty$ in order to calculate the mass. Indeed in this example

$$
\mathcal{J}=\frac{m}{4 \pi r^{2}} e^{01}
$$

and (46) is exactly analogous to Gauss' law in electrostatics. This similarity between the Maxwell 2-form field strength $F$ and $\mathcal{J}=d K$ for a Killing vector $\vec{K}$ was pointed out in [20].

\section{THE RELATION BETWEEN THE NOETHER CURRENT AND THE NOETHER 2-FORM}

In this section we expand further on the relation between the Noether current $j$ and the Noether 2-form $J$ for spacetime symmetries. Under a variation of the fields

$$
\delta L=d \theta
$$

on shell. The conventional Noether current is obtained from a symmetry generator $\mathcal{T}_{\mathcal{Q}}$ under which the Lagrangian is invariant on shell,

$$
\mathcal{T}_{\mathcal{Q}} L=d \theta=0
$$

and 


$$
\theta=* j
$$

For a Killing symmetry generated by a vector field $\vec{K}$

$$
\mathcal{L}_{\vec{K}} L=d i_{\vec{K}} L
$$

and

$$
d\left\{\theta(\vec{K})-i_{\vec{K}} L\right\}=0 .
$$

If $\theta(\vec{K})-i_{\vec{K}} L$ is $d$-exact we define $Q(\vec{K}) \bmod d$ via

$$
d Q(\vec{K})=\theta(\vec{K})-i_{\vec{K}} L .
$$

Under a genuine variation of the dynamical fields, which is not a diffeomorphism,

$$
\hat{\delta} L=d \hat{\theta}
$$

and, if $i_{\vec{K}} \hat{\theta}$ is $\hat{\delta}$-exact,

$$
i_{\vec{K}} \hat{\theta}=\hat{\delta} \mu(\vec{K}) .
$$

The 2-form $\mathcal{J}(\vec{K})$ (that is a 2 -form on $T^{*} \mathcal{M}$ ) is then defined via

$$
\hat{\delta} * \mathcal{J}=\hat{\delta} \mu-\hat{\delta} Q
$$

The definition of $\mathcal{J}$ is very different to the standard approach to the Noether current for space-time symmetries associated with a Killing vector. The standard approach assumes that the Lagrangian can be decomposed into a "geometrical" term and a "matter" term, e.g., for Einstein gravity

$$
L=-\frac{1}{16 \pi} R_{a b} \wedge * e^{a b}+L_{\text {Matter }} .
$$

If the metric is not dynamical only $L_{\text {Matter }}$ is considered. The energy-momentum tensor is defined by varying the metric in $L_{\text {Matter }}$, even if it is not dynamical. In terms of orthonormal 1-forms

$$
\delta_{e^{a}}\left(L_{\text {Matter }}\right)=\delta e^{a} \wedge \tau_{a},
$$

where

$$
\tau_{a}=T_{a b} * e^{b} .
$$

Conservation of energy-momentum can be expressed as

$$
D \tau^{a}=0 \Leftrightarrow D_{a} T_{b}^{a}=0,
$$

and we assume this is true on shell. Then for a diffeomorphism $\delta e^{a}=\mathcal{L}_{\vec{X}} e^{a}$ and

$$
\begin{aligned}
\delta_{\vec{X}} L_{\text {Matter }} & =\left(d i_{\vec{X}} e^{a}+i_{\vec{X}} d e^{a}\right) \wedge \tau_{a} \\
& =\left(d X^{a}+\omega^{a}{ }_{b} X^{b}-\left(i_{\vec{X}} \omega^{a}{ }_{b}\right) e^{b}\right) \wedge \tau_{a} \\
& =\left(D X^{a}\right) \wedge \tau_{a} \\
& =d\left(X^{a} \tau_{a}\right),
\end{aligned}
$$

where we have used $e^{b} \wedge \tau^{a}=T^{a b} * 1$ and $\omega_{a b}=-\omega_{b a}$. If $\vec{X}=\vec{K}$ is Killing then

$$
\delta_{\vec{K}} L_{\text {Matter }}=d\left(K^{a} \tau_{a}\right)=0
$$

on shell, and the Noether current

$$
* j=K^{a} \tau_{a}
$$

is conserved, $d * j=0$ (in components $j_{b}=K^{a} T_{a b}$ ).

This is clearly on a different footing to conservation of the 2-form, $d * \mathcal{J}=0$. The d-cohomology classes of $* j$ and $* \mathcal{J}$ are completely different-they carry different geometrical information.

\section{DISCUSSION}

Wald and Collaborator's description of diffeomorphic invariant theories (the generalization of Witten and Crnković description of Yang-Mills theories and general relativity) and the construction of symplectic structures and Noether charges fits naturally into a double complex structure, summarized in the commutative diagram (15). This mathematical structure is also relevant to quantum anomalies. The field variations $\delta$ include symmetry transformations of the classical action, and these can generate new terms if there is a quantum anomaly. The double complex not only gives a covariant description of phase space and the symplectic structure as well as classical invariants it also describes the cohomology of quantum anomalies.

The explicit example of the Schwarzschild metric shows how the timelike Killing vector generates the Noether charge associated with the mass. This can be calculated exactly on any sphere surrounding the event horizon, it is not necessary to perform the calculation at $r \rightarrow \infty$ (the only role the asymptotic regime plays is to furnish the correct normalization for the Killing vector, which is chosen to give $\frac{\partial}{\partial t}$ unit length only at $r \rightarrow \infty$ ). This is not in itself a new result-it was shown that the Noether charge correctly reproduces the ADM mass for asymptotically flat spacetimes in [8] - but the same formalism also correctly reproduces the Brown-York mass and the Bondi mass as well as the Henneaux-Teitelboim mass for a rotating black hole in asymptotically anti-de Sitter space-time, the details of these will be published elsewhere [12].

\section{ACKNOWLEDGMENTS}

The author acknowledges support by the Action MP1405 QSPACE from the European Cooperation in Science and Technology (COST). 
[1] A. Bilal, arXiv:0802.0634.

[2] L. Alvarez-Gaumé and P. H. Ginsparg, Ann. Phys. (N.Y.) 161, 423 (1985).

[3] C. Crnković and E. Witten, Covariant Description of Canonical Formalism in Geometrical Theories, in Three Hundred Years of Gravitation, edited by W. Israel and S. W. Hawking (Cambridge University Press, 1987).

[4] J. L. Friedman, Commun. Math. Phys. 62, 247 (1978).

[5] J. Lee and R. M. Wald, J. Math. Phys. (N.Y.) 31, 725 (1990).

[6] R. M. Wald, J. Math. Phys. (N.Y.) 31, 2378 (1990).

[7] R. M. Wald, Phys. Rev. D 48, R3427 (1993).

[8] V. Iyer and R. M. Wald, Phys. Rev. D 50, 846 (1994).

[9] R. Arnowitt, S. Deser, and C. W. Misner, Phys. Rev. 117, 1595 (1960).

[10] A. Komar, Phys. Rev. 113, 934 (1959).

[11] J. Katz, Classical Quantum Gravity 2, 423 (1985).
[12] B. P. Dolan, following Paper, Phys. Rev. D 98, 044010 (2018).

[13] J. D. Brown and J. W. York, Jr., Phys. Rev. D 47, 1407 (1993).

[14] H. Bondi, Nature (London) 186, 535 (1960).

[15] M. Henneaux and C. Teitelboim, Commun. Math. Phys. 98, 391 (1985).

[16] G. W. Gibbons, M. J. Perry, and C. N. Pope, Classical Quantum Gravity 22, 1503 (2005).

[17] K. Hajian and M. M. Sheikh-Jabbari, Phys. Rev. D 93, 044074 (2016).

[18] I. Papadimitriou and K. Skenderis, J. High Energy Phys. 08 (2005) 004.

[19] R. Bott and L. W. Tu, Differential Forms in Algebraic Topology, Graduate Texts in Mathematics (Springer, New York, 1982), Vol. 82.

[20] W. Simon, Gen. Relativ. Gravit. 17, 439 (1985). 